\title{
Entre destruction et édification: Ambivalence de la cité à la Renaissance
}

Muriel Cunin

\section{(2) OpenEdition \\ 12 Journals}

Édition électronique

URL : http://journals.openedition.org/shakespeare/1599

DOI : 10.4000/shakespeare.1599

ISSN : 2271-6424

\section{Éditeur}

Société Française Shakespeare

\section{Édition imprimée}

Date de publication : 1 mars 2011

Pagination : 43-54

ISBN : 2-9521475-7-4

\section{Référence électronique}

Muriel Cunin, "Entre destruction et édification : Ambivalence de la cité à la Renaissance », Actes des congrès de la Société française Shakespeare [En ligne], 28 | 2011, mis en ligne le 15 février 2011, consulté le 23 avril 2019. URL : http://journals.openedition.org/shakespeare/1599; DOI : 10.4000/ shakespeare.1599 


\section{Shakespeare et la Cité}

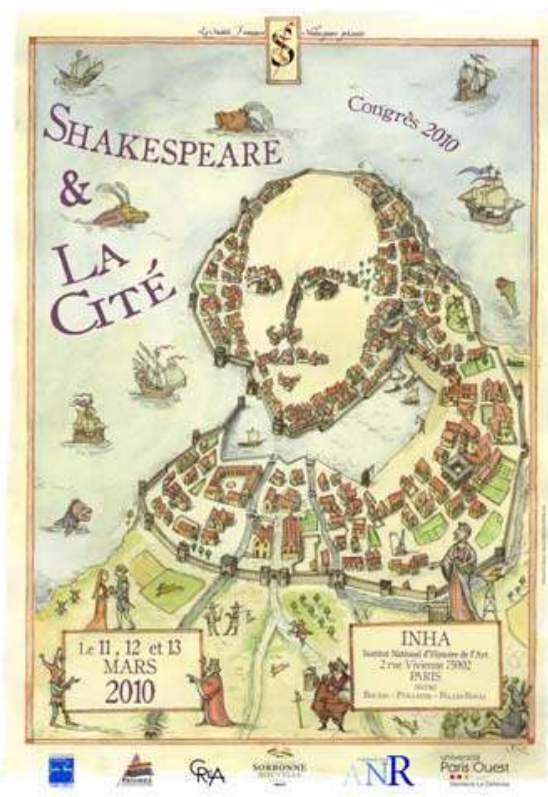

actes du Congrès

organisé par la

SOCIÉTÉ FRANÇAISE SHAKESPEARE

les 11,12 et 13 mars 10

textes réunis par

Pierre KAPITANIAK

sous la direction de

Dominique GoY-BLANQUET 
COUVERTURE :

Edouard Lekston 2010

conception graphique et logo

Pierre Kapitaniak

\section{(C) 2011 Société Française Shakespeare Institut du Monde Anglophone} Université de Paris III - Sorbonne Nouvelle 5 rue de l'École de Médecine 75006 Paris

www.societefrancaiseshakespeare.org réservés pour tous les pays 


\title{
ENTRE DESTRUCTION ET ÉDIFICATION : Ambivalence de la Cité À la Renaissance
}

\author{
Muriel CuniN
}

Parce qu'elle est soumise au temps, lui-même ambivalent, la cité est duelle et tendue entre destruction et édification. Le temps joue en effet un double rôle dans le processus architectural, comme le montre la passion de la Renaissance pour l'archéologie et pour le motif des ruines, à la fois sources de réflexion sur le passé et invitation à construire à partir de lui. Le lien de la cité à l'Histoire s'inscrit ainsi dans un processus éthique et politique qui reflète cette ambivalence fondamentale, illustrée par l'évolution de Londres à l'époque prémoderne.

Being subjected to time, which is itself ambivalent, the city is dual and partakes of both destruction and edification. Indeed, time plays a dual role in the architectural process, as testified by the Renaissance craze for archaeology and interest in ruins, which are both a source of reflection on the past and an incitation to use it as a basis for construction. The link of the city to History is part of an ethical and political process reflecting this fundamental ambivalence, illustrated by the evolution of London in the early modern age.

ultiple, protéiforme, changeante, la cité peut s'étudier sous plusieurs angles - politique, topographique, archéologique, architectural, matériel, subjectif, etc. - et se définir de diverses manières : comme la partie de la ville contenue entre des murailles (citadelle); comme un grand centre urbain ; comme polis ou civitas, communauté de citoyens censés observer les règles de la vie en société. Le civis, membre libre d'une cité s'oppose alors à l'hostis, hôte et étranger, comme la civilisation s'oppose à la barbarie. Mais quel que soit le point de vue adopté, la cité apparait duelle par nature. Parce que le temps s'inscrit au cœur même de l'élaboration de la cité, de son image, de sa mythologie ou de sa destruction, elle est marquée par une ambivalence très profonde, que Gail Kern Paster résume par la formule " an idea of two cities is almost always implicit in expressions of the idea of one city ${ }^{1}{ }^{~}$. L'idée même de cité est donc problématique en soi. Nous tenterons d'en présenter quelques enjeux en insistant sur la notion omniprésente d'ambivalence et en articulant point de vue historique et lecture mythique, inséparables à la Renaissance. Cet arrière-plan nous permettra ensuite de nous pencher plus particulièrement sur la situation de Londres, cité en pleine expansion à

\footnotetext{
${ }^{1}$ Gail Kern Paster, The Idea of the City in the Age of Shakespeare, Athens, Georgia, University of Georgia Press, 1985, p. 11. Notre premier paragraphe repose sur les pages 1113 de cet ouvrage.
} 
l'époque pré-moderne et tendue elle aussi entre destruction et édification.

\section{Dualité}

Dans La Cité de Dieu, saint Augustin oppose la cité terrestre, soumise au temps et à la destruction, à la cité céleste, idéale et édifiante. Mais l'opposition est moins simple qu'il n'y paraît : écrite en réaction au Sac de Rome (410 après JC), l'œuvre prend précisément Rome comme modèle de la cité humaine, faisant ainsi de la Ville Éternelle une forme d'archétype. La Cité de Dieu tient, malgré tout, de la laudatio urbis car c'est par rapport au modèle romain que s'élabore la cité céleste, qui le dépasse et représente une cité plus grande encore, au fondateur plus grand encore ${ }^{2}$. Rome fascine même ses détracteurs et l'Apocalypse de saint Jean, qui la présente sous les traits de la putain de Babylone, évoque aussi sa beauté et sa séduction : «Et la femme était vêtue de pourpre et d'écarlate, et toute dorée d'or, et de pierres précieuses, et de perles » (Apocalypse, 17,4). Dans l'Antiquité, cette ambivalence se retrouve au cœur même de la cité et Rome superpose les deux images en une sorte de coincidentia oppositorum. Pour les hommes de l'Antiquité, en effet, la cité idéale n'est pas la simple antithèse de la cité réelle mais l'idéal, réalisable en ce monde, vers lequel doit tendre la cité. La cité est donc à la fois ce qui ordonne (met en ordre) et ce qui est ordonné3, Rome est à la fois la cité terrestre et la Ville Éternelle, l'Urbs. Dans son évocation de la cité hellénique, Lewis Mumford évoque la même dualité entre terrestre et céleste :

Yes, the visible, tangible city was full of imperfections : the disorders of growth, the fermentations and secretions of life, the unburied refuse of outlived forms, not yet decently removed, the relics of rural ways not yet adjusted to the continued ordeals and challenges of urban life. Such a city might present momentary concentrations of significant form, as one climbed the steep path up the Acropolis at Athens and at last beheld the wide plain from an elevation of half a thousand feet; but one could not hope for prolonged order or sustained harmonies. Yet the inner carol of delight one might feel, when the rocks of the Acropolis fell away and one finally beheld the Parthenon itself, was perhaps all the keener for its contrast with the casual jumble and sprawl of the town below. [...] There is no better place to confront the paradoxical relation between the mind

\footnotetext{
${ }^{2}$ Voir Gail Kern Paster, op. cit., p. 12.
}

3 Gail Kern Paster, op. cit., p. 15. 
and the body through which it expresses itself, the social body that becomes a humanized landscape or a city, than in the Greek polis, above all in Athens ${ }^{4}$.

Cette même ambivalence se poursuit à la Renaissance, alors qu'une forme de proto-urbanisme se développe et que les plans de cités idéales, échos de l'harmonie céleste, prolifèrent. Mais la cité idéale n'est-elle pas une forme de ce que Mumford appelle « la régression vers l'utopie » ( « regression to utopia 5 ») ? Ne risque-t-elle pas, en éliminant toute ambivalence, d'éliminer également tout ce qu'il y d'humain dans une cité réelle ? Est-elle édifiante ou destructrice pour l'individu?

L'un des grands principes de l'architecture de la Renaissance est la firmitas (solidité), évoquée par Vitruve («il faut que les Fondemens soient creuzes dans le solide, ou jusqu'au solide autant que la grandeur de l'Édifice le requiert. Ils doivent estre bastis avec toute la solidité possible, sur le fond de la trenchée qui a esté faite ${ }^{6}$. ») et par Leon Battista Alberti7. Le temps en effet joue un double rôle dans le processus architectural. D'une part, il apparaît comme l'ennemi à combattre et le livre x du De re æedificatoria d'Alberti est intégralement consacré aux dégradations qu'il provoque et à la manière d'y remédier ; d'autre part, il est une garantie contre les erreurs si bien que l'architecte doit faire preuve de patience et de prudence et que sa qualité principale sera « la persévérance tenace et assidue [...] avec l'aide du temps ${ }^{8}{ }^{\prime}$. L'architecture est donc à la fois soumise au temps et capable de le soumettre. Lui-même est ambivalent, comme le rappelle cette gravure du Songe de Poliphile le représentant sous la forme d'une ronde de personnages, tous dotés de deux visages, l'un triste et l'autre gai (I, 4) ${ }^{9}$. La passion de la Renaissance pour l'archéologie participe de cette ambivalence, favorisée par l'image duelle de Rome. Le Moyen Âge met l'accent sur la Rome chrétienne, dégagée des errements du paganisme :

\footnotetext{
${ }^{4}$ Lewis Mumford, The City in History (1961), Harmondsworth, Penguin, 1966, p. 186-187. 5 Ibid., p. 200.

${ }^{6}$ Vitruve, Les Dix livres d'architecture (De architectura), trad. Claude Perrault (1684), Liège, Pierre Mardaga, s.d., III, 3, p. 84.

${ }^{7}$ Leon Battista Alberti, L'Art d'édifier (De re æxificatoria), trad. Pierre Caye et Française Choay, Paris, Éditions du Seuil, 2004, I, 9.

8 Ibid., II, 2, p. 101.

${ }^{9}$ Voir Muriel Cunin, Shakespeare et l'architecture. Nouvelles inventions pour bien bâtir et bien jouer, Paris, Honoré Champion, 2008 ; p. 256-257.
} 
On constate la présence de l'Antiquité, sans la nier : la Rome antique est le passé [...], le temps révolu, et ce sentiment, ajouté à l’idée qu'il y a un progrès chrétien, fait que l'on regarde le vestige comme la marque d'une époque moins éclairée religieusement, défaut que la ruine du temps punit $^{10}$.

À partir du XIVe siècle s'opère une certaine prise de conscience vis-à-vis du passé antique. Poggio Bracciolini, par exemple, souligne « la grandeur passée de Rome » tout en déplorant "sa misère présente ${ }^{11}$ » (De varietate fortunæ Urbis Romæ, 1447-48). Car les ruines n'ont pas seulement été abîmées par le temps, mais aussi par l'homme: de nombreuses œuvres ont été détruites, leur matériau (surtout le marbre) ayant été réutilisé pour des constructions récentes. On sait combien le Colisée, par exemple, a pu servir de carrière. De plus en plus d'érudits tentent alors de sauver ce qui peut encore l'être. Pour cela il faut, comme le souligne Alain Schnapp, « accepter que Rome soit mise à distance [...]. Il faut en fait admettre la coupure qui sépare le présent du passé, et donc traiter l'Antiquité comme un objet historique ${ }^{12} »$.

Pétrarque est l'un de premiers à protester contre le traitement que l'on fait subir aux monuments antiques, et des savants comme Boccace, Manuel Chrysoloras et Flavio Biondo lui emboîtent le pas. En littérature comme en peinture, le thème des ruines devient un véritable leitmotiv, de l'Italie au Nord de l'Europe - car l'Angleterre réformée est aussi victime des iconoclastes. Dans la lignée des Triomphes de Pétrarque (1470), la contemplation des ruines mène tout naturellement à une réflexion sur la vanité et la mutabilité de toute chose. D'ailleurs, comme le souligne Alain Schnapp, «la ville des morts, la Rome antique, n'est-elle pas plus grande que la ville des vivants ${ }^{13}$ ? » D'innombrables gravures représentent des ruines, d'innombrables textes les décrivent, du Songe de Poliphile de Francesco Colonna (1499) aux poèmes d'Edmund Spenser, The Ruines of Time et The Ruines of Rome, adaptation des Antiquités de Rome de Du Bellay. Ces textes se veulent édifiants : loin d'être uniquement des memento mori,

\footnotetext{
10 Martine Furno, «La Descriptio Urbis Romæ dans l'histoire du latin et de la culture humaniste ", in Leon Battista Alberti, Descriptio Urbis Romæ, éd. Martine Furno et Mario Carpo, Genève, Droz, 2000, p. 109.

${ }^{11}$ Martine Furno, ibid., p. 112.

12 Alain Schnapp, La Conquête du passé. Aux origines de l'archéologie, Paris, Éditions Carré, 1993, p. 133.

13 Ibid., p. $147-148$.
} 
ils sont également une invitation à tirer les leçons du passé et à construire à partir de lui. Spenser, afin de préserver la mémoire des lieux qu'il évoque, se livre à une reconstruction mentale accomplie grâce à la figure de l'ekphrasis. Il rend d'ailleurs hommage à William Camden, le fondateur de la tradition des antiquaires anglais. Cette importance de la description, on la retrouve aussi bien chez Chrysoloras que chez Colonna ou chez Poggio Bracciolini. Préserver les ruines, c'est préserver la mémoire, et donc assurer l'avenir : « Thy lord shall never die, the whiles this verse / Shall live and surely it shall live forever », écrit Spenser dans The Ruines of Time. Le mot « monument » ne vient-il pas de monere, à la fois rappeler et avertir ? On songe ici au récit que fait Vitruve lorsqu'il relate l'origine des caryatides :

Leur ville [Carie, dans le Péloponnèse] ayant esté prise \& ruinée, \& tous les hommes mis au fil de l'épée, les femmes furent emmenées captives \& [...] on ne permit pas aux Dames de qualité de quitter leurs robes accoûtumées, ny aucun de leurs ornemens, afin que non seulement elles fussent une fois menées en triomphe, mais qu'elles eussent la honte de s'y voir en quelque façon mener toute leur vie, paroissant toujours au mesme état qu'elles estoient le jour du triomphe, \& qu'ainsi elles portassent la peine que leur ville avoit meritée. Or pour laisser un exemple éternel de la punition que l'on avoit fait souffrir aux Cariates, \& pour apprendre à la posterité quel avoit esté leur châtiment, les Architectes de ce temps-là mirent au lieu de colonnes, ces sortes de Statuës aux Édifices publics ${ }^{14}$.

La ville renaissante s'inscrit ainsi dans un double processus de mémoire et d'édification qui souligne son lien à l'Histoire. En s'inspirant de l'architecture antique, l'architecture de la Renaissance contribue à remplacer une approche purement livresque des ruines par un réel travail sur le terrain. La mémoire est liée à l'architecture et à l'urbanisme, par exemple à travers la pratique de l'ars memoriæ. Décrite par Cicéron (De oratore), par Quintilien (Institutio oratoria) et par l'auteur anonyme de la Rhetorica ad Herennium, cette technique consiste à mettre en place une série de «lieux » (loci memoriæ), en général les pièces d'un bâtiment ou les rues d'une ville, dans lesquels l'orateur place des personnages (imagines) correspondant aux éléments à mémoriser. Pour se souvenir, il suffit ensuite de parcourir mentalement les pièces du bâtiment ou les rues de la ville. Dans son $D e$

\footnotetext{
14 Vitruve, Les Dix Livres d'architecture, I, 1, p. 4.
} 
memoria $(1553)^{15}$, Guglielmo Grataroli utilise sa ville natale pour créer des loci dont la description n'est pas sans rappeler les ouvrages d'archéologie et les guides urbains de la Renaissance : se souvenir, c'est lire la ville et inversement. En outre, Grataroli pare la mémoire de vertus édifiantes en faisant d'elle la mère de la sagesse ${ }^{16}$. Le lien entre cité, mémoire, et édification est ainsi confirmé.

\section{Polis et politique}

Édifiée et fortifiée par le travail de mémoire, la citadelle de l'âme est mieux parée pour résister aux assauts des vices. Le lien de la cité à l'Histoire s'inscrit donc tout naturellement dans un processus éthique et politique, la cité de la Renaissance cherchant véritablement à répondre à l'idéal de la polis grecque. Le titre latin du traité d'Alberti, De re ædificatoria - que l'on décrit trop souvent, de manière un peu hâtive, comme une réécriture de celui de Vitruve - est révélateur et va plus loin que le «de architectura » de son illustre prédécesseur ${ }^{17}$, comme le souligne Giulio Carlo Argan :

Alberti dit que tous les arts convergent et que leur fin commune est [...] la vie qui se déroule dans une cité bien ordonnée. Ce qu'Alberti veut définir et expliquer dans le De re ædificatoria, c'est la forme de l'espace urbain par rapport à la forme de l'espace naturel. Est urbain l'espace où vit une société organisée, où les techniques du travail humain s'élèvent à la dignité de science, où se prennent des décisions politiques et s'organisent des actions destinées à produire des effets lointains et durables : en un mot, l'espace de la ville est l'espace de l'Histoire ${ }^{18}$.

Mais les hommes de la Renaissance se heurtent fréquemment au problème du meurtre de Rémus par Romulus, qui contredit l'idée humaniste selon laquelle la fondation d'une ville est l'acte héroïque par excellence. Dès Plutarque, on cherche des alternatives à cet acte

\footnotetext{
15 Traduit en anglais 1562 par William Fulwood sous le titre The Castel of Memorie, et en français dès 1555 par Étienne Coppé sous le titre Des préceptes et moyens de recouvrer, augmenter et contregarder la mémoire.

16 "Wisedome is the doughter of memorye ", trad. William Fulwood, op. cit., sig. B5 $\mathrm{r}^{\circ}$.

17 Voir Françoise Choay, La Règle et le Modèle. Sur la théorie de l'architecture et de l'urbanisme, Paris, Éditions du Seuil, 1980 et Roy Eriksen, The Building in the Text. Alberti to Shakespeare and Milton, University Park, Pa, Penn State Press, 200o, p. 49-77.

18 G. C. Argan, «Le traité De re æedificatoria », in Giulio Carlo Argan et Rudolf Wittkower, Perspective et histoire au Quattrocento, trad. Jean-Jacques Le Quilleuc, Marc Perelman et Fra Marcello, Paris, Éditions de la Passion, 1990, p. 88.
} 
sanglant, et Virgile préfère désigner Énée comme fondateur de Rome, tandis que les traités de poétique reprennent à l'envi les mythes d'Amphion et d'Orphée :

Amphion and Orpheus, two Poets of the first ages, one of them, to wit Amphion, builded up cities, and reared walles with the stones that came in heapes to the sound of his harpe, figuring thereby the mollifying of hard and stonie hearts by his sweete and eloquent persuasion. And Orpheus assembled the wilde beasts to come in heards to harken to his musicke, and by that meane made them tame, implying thereby, how by his discreete and wholsome lesons uttered in harmonie and with melodious instruments, he brought the rude and savage people to a more civill and orderly life, nothing, as it seemeth, more prevailing or fit to redresse and edifie the cruell and sturdie courage of man then it ${ }^{19}$.

Selon le récit associé à la fondation de la cité, celle-ci symbolisera donc l'harmonie et la civilité ou, au contraire, la corruption et la sauvagerie.

Londres au XVI ${ }^{\mathrm{e}}$ siècle illustre parfaitement cette inscription de la cité dans l'histoire et la politique, et l'ambivalence qui en découle. Le paysage urbain est fortement modifié par la Réforme, dont les effets sont eux-mêmes duels : destruction d'une part, reconstructions et innovations de l'autre. Les déprédations liées à la Réforme sont suffisamment importantes pour qu'Élisabeth $\mathrm{I}^{\mathrm{re}}$ publie une proclamation le 19 septembre 1560 " against breakinge or defacing of monuments of antiquitie, beyng set up in Churches or other publique places for memory, and not for supersticion ». Mais la destruction est aussi parfois un premier pas vers la reconversion : les matériaux sont réutilisés, les bâtiments transformés, et pour Maurice Howard «the fate of monastic sites in the urban environment provides one of the most emphatic pieces of evidence of continuity between their state in medieval times and their post-dissolution conversion ${ }^{20}$ ». Une forme de continuité avec le passé s'établit ainsi, tout en donnant naissance à certaines nouveautés. Londres devient un palimpseste ${ }^{21}$. Des cloîtres sont transformés en cours ou en loggias, par exemple. À ces conversions s'ajoute la superposition du gothique vernaculaire et des ornements classiques importés par les amateurs ainsi que par les artisans des débuts du règne d'Henri viII et du règne d'Édouard vI,

19 Voir Gail Kern Paster, op. cit., p. 9-11.

${ }^{20}$ Maurice Howard, The Early Tudor Country House. Architecture and Politics 1490-1550, Londres, George Philip, 1987, p. 29.

${ }^{21}$ Voir Maurice Howard, The Building of Elizabethan and Jacobean England, New Haven \& Londres, Yale University Press, 2007, p. 18 
tandis que deux discours opposés se superposent: pour certains, le gothique est rude et barbare (Wotton parle de «reliques of [a] barbarous age $\left.\mathrm{a}^{22} »\right)$, pour d'autres, au contraire, il représente la tradition vernaculaire et l'esprit même de l'Angleterre ; pour certains, le classicisme est associé à l'idolâtrie et au paganisme, pour d'autres, il est au contraire une saine alternative au gothique, catholique et idolâtre. Les descriptions de la ville se multiplient, oscillant entre chorographie et panégyrique (William Harrison, William Camden, John Norden, John Stow, Thomas Platter, John Speed, etc.). De grands changements sont à l'œuvre: de nouvelles catégories de bâtiments sortent de terre (les théâtres, le Royal Exchange en 1570, le New Exchange en 1608) tandis que la vieille Tour de Londres, chargée d'histoire et plus ou moins mythifiée, permet aux Londoniens de jeter un pont entre leur cité et l'Antiquité, via Jules César, à qui la légende attribue la construction d'origine. Source à la fois de fierté nationale et de peur liée à la réputation sinistre qui se développe autour d'elle au $\mathrm{XVI}^{\mathrm{e}}$ siècle, la Tour est un symbole ambivalent, comme le montre une étude récente de Kristen Deiter ${ }^{23}$. Enfin, l'apparente juxtaposition de la Cité et de la Cour et les relations complexes entre ces deux pôles ajoutent à l'impression générale de dualité (on notera au passage que vers 1609 on se met à désigner la Cour par l'expression «the Town ») $)^{24}$. Pourtant, le théâtre fait partie de ce qui lie Cour et Cité, malgré leurs divergences :

The early modern ambivalence towards the city is nowhere more marked than in attitudes to the theatre. On the one hand, theatre is promoted by apologists like Heywood as the proper ornament of any metropolis that seeks to emulate the splendours of Greece and Rome; on the other, it is identified by its enemies - and sometimes, surprisingly, even by its own practitioners, as a place of disorder, a bastion of the unruly mob, a leveller of proper distinctions, and a source of moral and physical disease ${ }^{25}$.

La Cour comme la Cité ont besoin de la pageantry et la recherche récente tend à insister davantage sur l'idée de la fluidité entre ces deux

\footnotetext{
${ }^{22}$ Henry Wotton, The Elements of Architecture, Londres, 1624 (STC 26011), p. 51.

23 Kristen Deiter, The Tower of London in English Renaissance Drama. Icon of Opposition, New York \& Londres, Routledge, 2008.

24 Voir Janette Dillon, Theatre, Court and City. 1595-1610. Drama and Social Space in London, Cambridge, CUP, 2000.

25 Michæl Neill, Issues of Death, cité par Gail Kern Paster, op. cit., p. 32-33.
} 
espaces que sur leur séparation : Ian W. Archer, par exemple, montre que les événements royaux tels que funérailles et couronnements renforçaient les liens entre les deux car les pageants étaient payés par la Cité et les vêtements et étoffes par la Couronne, et que ces événements avaient un fort impact sur l'économie de la Cité ${ }^{26}$. De la même manière, la séparation entre la Cité intra muros et ses alentours s'efface à l'époque pré-moderne, comme le souligne John Speed dans son Theatre of the Empire of Great Britain (1611) :

This London, as it were disdaining bondage, hath set herself, on each side, far without the walls, and left her west gate in the midst, from whence with continual buildings (still affecting greatness) she hath continued her streets unto a king's palace, and joined a second city to herself [...]. No walls are set about this city, and those of London are left to show rather what it was than what it is [...]. The wealth of this city [...] grows from the revenues and harvest of her south-bounding Thames. [...] And from London her channel is navigable, straitened along with meadowing borders, until she taketh her full liberty in the German Seas. Upon this Thames the ships of Tharsis seem to ride, and the navy, that rightly is termed the Lady of the Sea, spreads her sail, whence twice with lucky success hath been accomplished the compassing of the universal globe ${ }^{27}$.

Ce que Speed décrit ici, c'est une cité qui devient une métropole, s'étend au-delà des vieilles murailles médiévales, rejoint Westminster, absorbe les espaces verts et les villages alentours, et dont le fleuve permet de faire le tour du monde. Il y a, comme le remarque Chris R. Kyle, " two Londons - the walled, corporate city and the metropolitan expanse $^{28} »$.

La population de Londres explose au Xvi siècle, passant de 50 ooo habitants en 1509 à 500000 en 1660. Cette inflation, due essentiellement à l'arrivée massive de personnes attirées par la ville et venant y chercher une situation ou des divertissements, entraîne de sérieux problèmes de surpopulation et une croissance anarchique des habitations, contre laquelle Jacques $\mathrm{I}^{\mathrm{er}}$ se battra (en vain) pendant tout son règne, publiant proclamation sur proclamation pour tenter de

\footnotetext{
${ }^{26}$ Ian W. Archer, «City and Court Connected: The Material Dimensions of Royal Ceremonial, ca. 1480-1625 ", in The Places and Spaces of Early Modern London, numéro spécial de Huntington Library Quarterly, vol. 71, nº 1 , mars 2008, p. 157-179.

${ }^{27}$ Cité dans Lawrence Manley éd., London in the Age of Shakespeare: An Anthology, Londres \& Sydney, Croom Helm, 1986, p. 43.

28 Chris R. Kyle, "Afterword : Remapping London », in The Places and Spaces of Early Modern London, op. cit., p. 244.
} 
ramener un peu d'ordre dans ce chaos urbain. Des extensions poussent sur les façades, surplombant les rues au point, parfois, de boucher presque complètement la vue du ciel, des baraques clandestines prolifèrent dans les arrière-cours, les familles s'entassent avec leurs visiteurs dans des logements de plus en plus exigus que l'on subdivise pour pouvoir en louer une partie. Les conséquences sont évidentes : problèmes de salubrité, d'approvisionnement en eau, risques d'incendie, développement de la petite criminalité, de la promiscuité, de la prostitution et des risques de fornication. La Cité s'en émeut, comme le montrent les études de cas menées par Lena Cowen Orlin dans un travail récent consacré aux logements temporaires à Londres $^{29}$, et la méfiance des autorités vis-à-vis des «inmates » (visiteurs temporaires) renvoie clairement à l'opposition étymologique entre civis et hostis évoquée plus haut. On est loin de « la vie dans une cité bien ordonnée » rêvée par Alberti. Une première proclamation, publiée le 7 juillet 1580 par Élisabeth $\mathrm{I}^{\mathrm{re}}$ " for one Family to a House », décrit très clairement la situation :

Where there are such great multitudes of people brought to inhabit in small rooms, whereof a great part are seen very poor, yea, such as must live of begging or by worse means, and they heaped up together, and in a sort smothered with many families of children and servants in one house or small tenement, it must needs follow (if any plague or popular sickness should by God's permission enter amongst those multitudes) that the same would not only spread itself and invade the whole city and confines [...] but would be also dispersed through all other parts of the realm...

Reprenant les mêmes idées à plusieurs reprises, Jacques $\mathrm{I}^{\mathrm{er}}$ insiste en outre sur la nécessité d'utiliser la brique et de renoncer au bois en raison de pénuries et de risque d'incendies. Il déplore également le manque d'uniformité des façades de Londres ( $1^{\mathrm{er}}$ mars 1605$)$ et finit par s'impatienter dans cette proclamation du 16 juin 1615 (qui ne sera pas la dernière) :

It is more than time that there be an utter cessation of further new buildings, lest the surcharge and overflow of people do bring upon our said City infinite inconveniences, which have been so often mentioned, both in our former proclamations and in the decrees of our High Court of Star Chamber, as it were in vain to repeat them. [...] [F]or further

29 « Temporary Lives in London Lodgings », in The Places and Spaces of Early Modern London, op. cit., p. 219-242. 
addition of private buildings we are fully resolved that this our Royal Proclamation, and further and peremptory commandment, shall be the furthest and utmost period and end of them ; only as to private houses, we could desire and wish [...] that we whom God hath honoured to be the first king of Great Britain, mought be able to say in some proportion, that we had found our City and suburbs of London of sticks, and left them of brick, being a material far more durable, safe from fire, beautiful, and magnificent.

Par ailleurs, le roi tente également de renvoyer à la campagne les membres de la gentry qui accourent à Londres, aggravant les problèmes de logement et mettant en danger la tradition de l'hospitalité associée aux « country houses » :

It is the fashion of Italy [...] that all the gentry dwell in the principal towns, and so the whole country is empty: even so now in England, all the country is gotten into London, and the whole country be left waste. [...] Let us in God's name leave these idle foreign toys, and keep the old fashion of England : for it was wont to be the honour and reputation of the English nobility and gentry, to live in the country, and keep hospitality ${ }^{30}$.

Comme Rome avant elle, Londres passe du statut de cité à celui de métropole. Comme Rome avant elle, Londres devient un monde: "London is not said to be in England, but rather England to be in London $^{31}$ ». Étymologiquement, la métropole est une ville-mère, mais Londres est une mère ambivalente, aimante et dévoratrice, absorbant les espaces verts, les faubourgs et les villages autour d'elle, et créant ainsi un espace à la fois horizontal, fluide, en continuelle expansion, et vertical, celui du palimpseste qui révèle le rôle double du temps, destructeur et édifiant.

L'importance de la pageantry montre que les arts et les lettres ont un rôle à jouer dans la cité. En faisant de la ville un théâtre, l'artiste contribue à l'édification politique, sinon morale, de ses concitoyens : " if a man shoulde say well, he could not better terme the citie of London that time, than a stage wherin was showed the wonderfull spectacle of a noble hearted princesse toward her most loving people ». Ainsi est décrite Londres le jour de l'Entrée d'Élisabeth Ire l'année de son couronnement. Ces spectacles de rue à l'architecture éphémère

$3^{0}$ Discours prononcé à la Star Chamber en 1616, cité par Janette Dillon, op. cit., p. 20.

${ }^{31}$ Thomas Platter, Travels in England (1599), cité dans Lawrence Manley éd., op. cit., p. 38 . 
présentent une vision idéale de l'architecture urbaine et transforment la cité en théâtre d'un pouvoir idéal. Ainsi Nicolas v élabore-t-il, durant son court règne (1447-1455) un projet de restauration, d'assainissement et de restructuration de Rome, persuadé que le spectacle urbain a son rôle à jouer dans l'édification des foules :

La masse de la population est ignorante et non lettrée ; elle est privée de culture ; il est bien qu'elle s'entende affirmer par les grands et par les érudits que l'autorité de l'Église est immense [...]. Pourtant elle a besoin d'être frappée par la vue de spectacles grandioses ; autrement, appuyée sur des bases instables, sa conviction finirait, le temps passant, par se réduire à rien ${ }^{32}$.

Même si l'on est encore loin de la cité idéale, l'Italie fournit tout de même des modèles d'urbanisme, mais c'est d'abord en peinture - grâce aux peintres perspectivistes - et en marqueterie, puis au théâtre - par le biais de la scénographie - et dans les jardins que l'on applique le principe de la perspective centrale. Ensuite seulement la perspective intervient dans le décor urbain, en trompe-l'œil : pendant les fêtes, on bâtit la cité idéale grâce à des constructions éphémères, mais aussi en déguisant les façades. Les architectes, contraints par les irrégularités du tissu urbain, créent un ordre factice et donnent naissance à un espace fictif, théâtral et irréel. C'est le type de décor idéal mais immatériel que l'on retrouve dans les décors d'Inigo Jones pour les masques de Cour : peu à peu, alors que le mur de fond de scène s'ouvre sur l'infini et que le trompe-l'œil signe la dissolution progressive des formes architecturales, la représentation de la cité idéale glisse vers l'immatérialité et la dissolution des formes.

Muriel CUNIN

Université de Limoges

\footnotetext{
32 Paroles prononcées par Nicolas $\mathrm{v}$ juste avant sa mort, d'après sa biographie par G. Manetti. Cité par Manfredo Tafuri, Architecture et humanisme de la Renaissance aux réformes, trad. Odile Seyler et Henri Raymond, Paris, Dunod, 1981, op. cit., p. 57. Tafuri décrit en détail le programme de Nicolas v, p. 54-57.
} 